\title{
Surgical Correction for Late Postoperative Subluxation of a Diffractive Optic Multifocal IOL, Capsule Bag and Capsule Tension Ring Complex: Case Report
}

\author{
Samuel Masket (iD)
}

Received: December 17, 2021 / Accepted: February 1, 2022 / Published online: February 26, 2022

(c) The Author(s) 2022

\begin{abstract}
Diffractive optic intraocular lenses (IOLs) require near perfect centration for best performance. A patient with a multifocal IOL and pseudoexfoliation developed late marked subluxation of the capsule bag, capsule tension ring and multifocal IOL complex. The surgical challenge was to fixate and recenter the diffractive optic IOL in order to restore normal quality vision; traditional surgical methods are not adequate for repositioning IOLs of this nature. A unique surgical plan that included three-point radially oriented suture fixation of an existing standard capsule tension ring (CTR) was conceived and was successful in achieving the patient's goal of maintaining spectacle independence by returning his vision to previous levels with a well-centered multifocal IOL.
\end{abstract}

Keywords: Adjustable slip knot; Diffractive optic IOL; Malpositioned IOL; Multifocal IOL; Pseudoexfoliation; Radial suturing; Scleral

Supplementary Information The online version contains supplementary material available at https:// doi.org/10.1007/s40123-022-00478-y.

S. Masket $(\square)$

Stein Eye Institute Geffen School of Medicine Los Angeles, Advanced Vision Care, Suite 911, 2080 Century Park East, Los Angeles, CA 90067, USA e-mail: avcmasket@aol.com suture fixation; Subluxated capsule bag/IOL/ CTR complex

\section{Key Summary Points}

Diffractive optic intraocular lenses (IOLs) require near perfect centration for best vision.

Late optic decentration may occur in cases with progressive zonulopathy.

Placing a capsule tension ring (CTR) at primary cataract surgery may be useful in cases at risk for late zonulysis.

3 Point scleral suture fixation of the CTR with adjustable suture tension can provide excellent recentering of the optic centration after late zonulysis.

\section{DIGITAL FEATURES}

This article is published with digital features, including a video, to facilitate understanding of the article. To view digital features for this article, go to https://doi.org/10.6084/m9. figshare.19097432. 


\section{INTRODUCTION}

Accurate centration of diffractive optic intraocular lenses (IOLs) (multifocal, trifocal and extended depth of focus) is essential to ensure high-quality postoperative visual function. Decentration of such an IOL from the visual axis can allow passage of central light through the diffractive rings but not the center of optic zone, resulting in higher-order aberrations and reduced vision quality $[1,2]$. This concern can create a conundrum for the cataract surgeon when confronted with a patient with pseudoexfoliation (PEX) (or other potential causes of progressive zonulopathy) who is desirous of a diffractive optic IOL but has no sign of zonulopathy at the time of cataract surgery. This situation creates a potential "time bomb" with a fuse of unknown length, as zonulysis with IOL subluxation may occur months to years later. Patients with such conditions are often dissuaded from receiving diffractive optic IOLs; however, that approach denies the patient who is highly motivated for spectacle independence. In cases where IOLs exhibit late decentration, lasso suture fixation of the two haptics may be employed to recenter the IOL, although that method is unlikely to provide the necessary precision required for diffractive optic centration. Another consideration is to explant the entire bag complex and fixate a monofocal IOL to the angle, iris, or sclera. But, in the interest of salvaging the diffractive optic IOL and restoring the previously satisfactory visual result of cataract surgery, a potentially useful strategy is to implant a standard or modified capsule tension ring (CTR) at initial cataract surgery for scleral suture fixation later should the capsule bag/ IOL/CTR (bag complex) decenter in the future [3]. This approach involves three-point, adjustable tension, radially oriented, suture fixation of the CTR to the sclera, affording excellent centration of the bag complex, necessary for proper performance of diffractive optic IOLs. The following case report, where patient consent was received for the publication of the data, is illustrative of this unique technique.

\section{CLINICAL FINDINGS}

A 75-year-old man was referred with significant bilateral mixed nuclear and cortical cataracts. He was highly motivated for spectacle independence with multifocal IOLs. Pseudoexfoliation was noted clinically for the left eye (LE) only; however, there was no evidence for phacodonesis, subluxation, or other sign of overt zonulopathy. Intraocular pressure (IOP) was normal at $13 \mathrm{mmHg}$ bilaterally. No further ocular abnormalities were noted, although the patient was maintained on oral tamsulosin for prostatism and exhibited poor pharmacologic pupil dilation for the LE; in response to the patient's need for tamsulosin, atropine sulfate $1 \%$ was administered topically 2 days prior to surgery to preclude intraoperative "floppy iris syndrome" [4]. At surgery, initially for the right eye (RE), a single-piece multifocal IOL (Alcon ReSTOR SN6AD1) was implanted after routine phacoemulsification. At surgery for the LE, however, a Malyugin ring (MST) was employed for small pupil management, and after uncomplicated phacoemulsification, a standard CTR (AMO STBL 12US) was placed within the capsule bag followed by a multifocal IOL (Alcon ReSTOR SN6AD1) that matched the fellow RE. The IOL and capsule bag were centered and stable.

After cataract surgery, the patient experienced excellent spectacle-free vision bilaterally, with uncorrected distance visual acuity (UCDVA) of $2025+2$ and uncorrected near visual acuity (UCNVA) of J1- for 5 years, but returned for care thereafter, reporting a recent onset of reduced vision for the LE; at this moment UCDVA was recorded at 20/60-2. As can be noted in Fig. 1, the bag complex had subluxated inferiorly with the superior edge of the IOL decentered to just below the mid-pupil; there was no anterior vitreous prolapse. There were no abnormalities observed for the uninvolved RE at this time. Given that the patient was satisfied with the vision achieved with the IOL for the LE prior to subluxation, a surgical plan was devised to retain rather than remove the bag complex. Consideration was given to "lasso" suture fixation of the IOL haptics to the 
sclera; however, even under a "best case" scenario it would be unlikely that perfect centration could be achieved. Rather, a plan was conceived to fixate only the CTR to the sclera, bypassing the IOL haptics, employing three points of fixation, each 120 degrees apart for potentially stable, centered fixation.

\section{THERAPEUTIC INTERVENTION}

At surgery (see Video) the limbus was marked in three positions, $120^{\circ}$ apart, and small adjacent peritomies fashioned. As the pupil dilated poorly, iris retractors (MST) were positioned where needed and the sclera was marked $3.5 \mathrm{~mm}$ posterior to the limbus for the posterior entry of the suture pathway. In this case a 10-0 polyester suture (Alcon, no longer available) was utilized, and the needle passed through the sclera, underneath the capsule bag and CTR, then through the capsule bag into the anterior chamber, avoiding the IOL haptic (Fig. 2). For the present case, 10-0 polyester suture was employed, but is no longer available. Current best options include use of 9-0 polypropylene or 8-0 Gore-Tex [polytetrafluoroethylene (ePTFE)] (Gore Industries). The needle was then docked in a 25-gauge bent hypodermic needle that entered the anterior chamber from an opposing paracentesis. Finally, the needle was turned around to re-enter the chamber through the same paracentesis (with care taken to avoid capturing any corneal fibers) and was docked into a 25-gauge bent hypodermic needle that pierced the sclera $1.5 \mathrm{~mm}$ posterior to the limbus and anterior to the capsule bag in the same meridian as the initial pass, completing a radially oriented "lasso" suture around the CTR.

The suture process was carried out in identical fashion in all three locations. Afterwards the iris retractors were removed, the OVD aspirated, a miotic instilled and IOP reestablished at physiologic levels. At this point the sutures were tied with adjustable slip knots and gradually tensioned until the IOL was centered, employing the diffractive rings and the pupil margin as guides. Next the knots were completed and rotated into the sclera and the peritomies

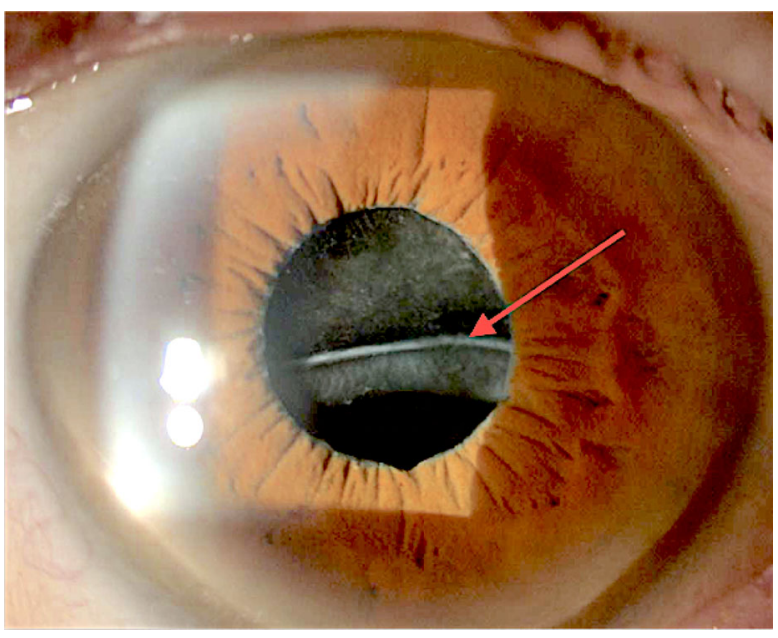

Fig. 1 Subluxated capsule bag/IOL/CTR complex LE noted preoperatively. Arrow indicates superior edge of IOL within the capsule bag. Note poor pupil dilation, Soemmerring's ring, and obvious pseudoexfoliative changes at the pupil margin

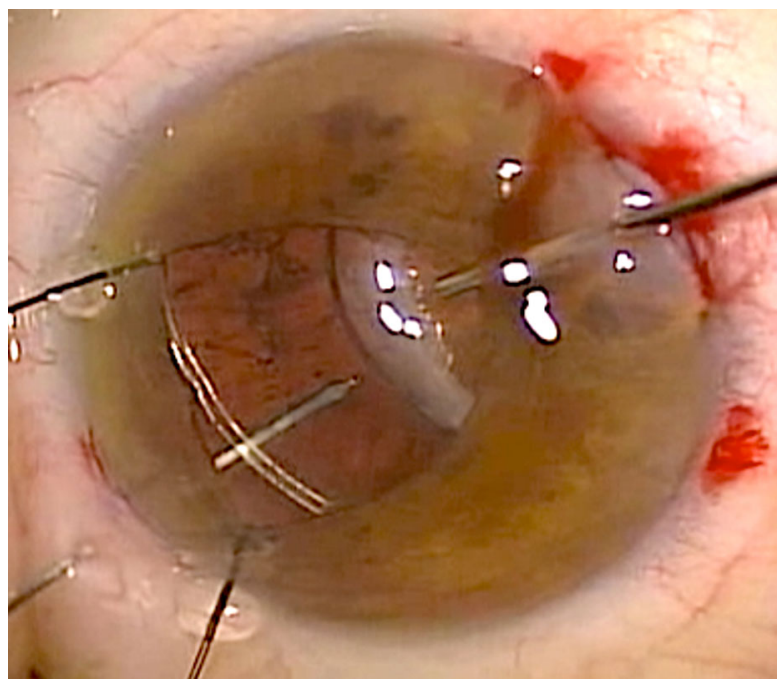

Fig. 2 The suture needle (from another surgical case) is passed from the sclera through the pars plana underneath the capsule bag (from the left) and pierces the capsule central to the CTR and enters the anterior chamber where it can be docked into a hypodermic needle or cannula (on the right) as in this case. After docking it is turned around and passed through the paracentesis and then docked into a needle that pierces the sclera anterior to the capsule bag complex, creating a lasso around the CTR. Note that the iris retractors are strategically placed to create exposure where most necessary 

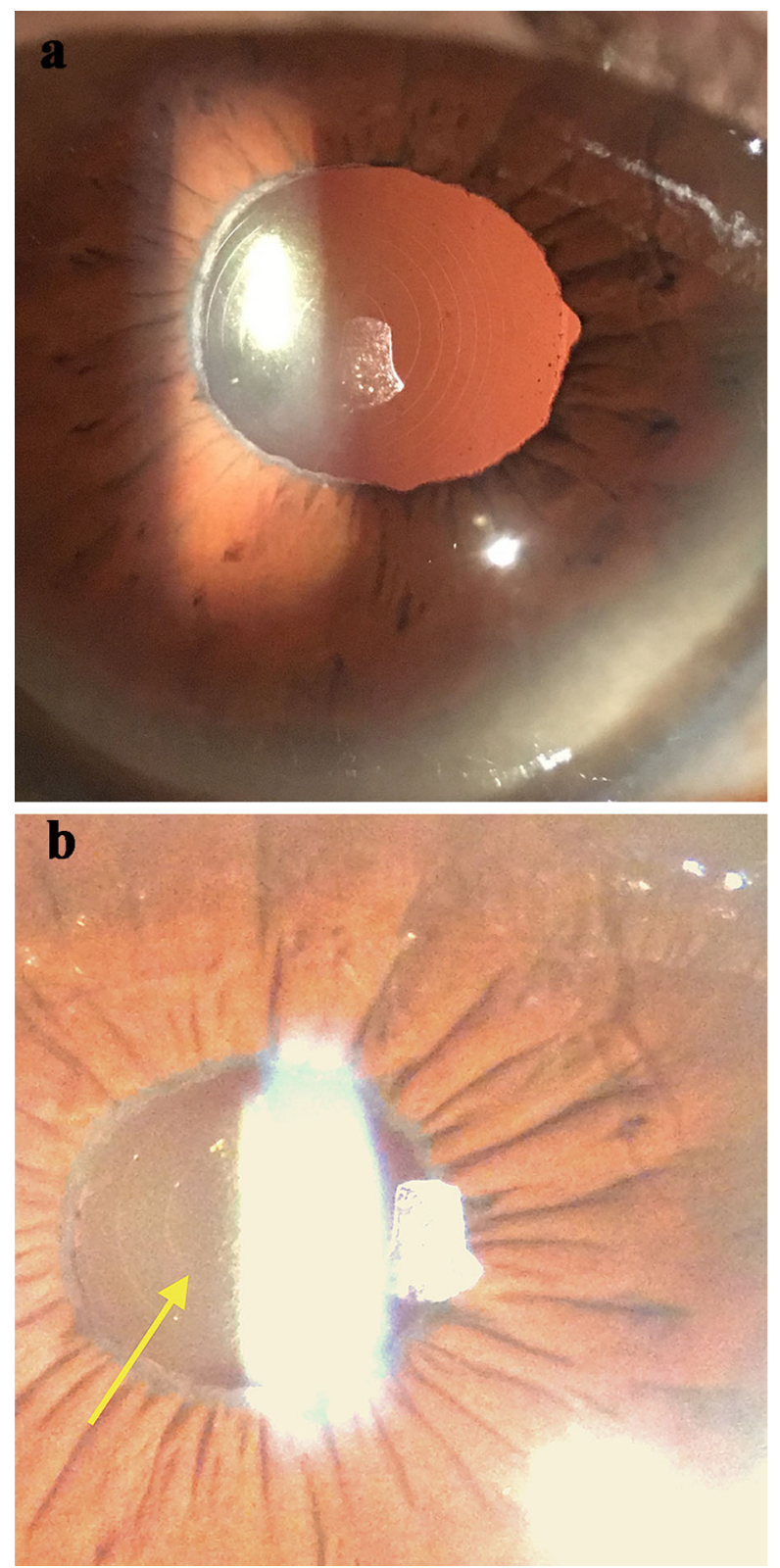

Fig. 3 Postoperative view of recentered IOL with pupil dilation (A). With physiologic pupil (B) one can note that the central ring of the diffractive optic IOL (arrow) is perfectly centered with the visual axis and concentric with the pupil

closed. I employed fibrin adhesive rather than suture closure of the conjunctiva.

Postoperatively, the IOL was noted to be perfectly centered and the vision restored to pre-subluxation acuity and quality (Fig. 3). At

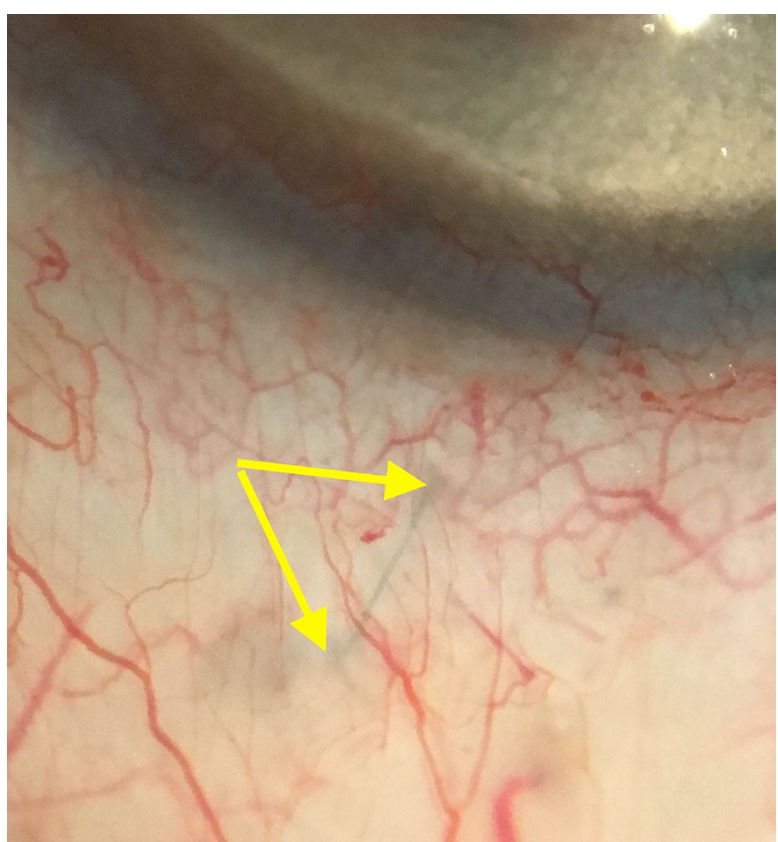

Fig. 4 10-0 Polyester suture pathway is radially oriented (arrows) to prevent torque of the CTR-bag complex. The knot is buried within the sclera

last examination, 5 years hence, the optic remained on center and the capsule bag complex stable with no evidence for pseudophacodonesis or tilt.

\section{DISCUSSION}

As can be noted in Fig. 3, excellent IOL centration can be achieved with the described method that includes radially oriented suture passage to avoid torque of the bag complex (Fig. 4), suture fixating only the CTR and not the IOL haptics, adjustable suture tensioning at physiologic IOP, and employing three-point fixation for centration and stability. This concept allows the option of implanting diffractive optic IOLs in patients with PEX or other causes of progressive zonulopathy as long as a CTR or modified CTR is placed at the original cataract surgery. The question then becomes whether a CTR should be used routinely in cases with PEX, as has been previously suggested [5]. However, as the current case suggests and as experience 
corroborates, a CTR will not prevent progressive zonulysis and late capsule bag complex subluxation [3, 6-8]. Nevertheless, when considering a diffractive optic IOL, as in the present case, routine use of a CTR may be potentially useful for management of late subluxation.

Three-point fixation of a subluxated CTR was reported earlier in two separate reports $[3,9]$. Ahmed et al. [3] described the concept for two cases, whereas Deka et al. [9] presented a single patient case. For the latter, the bag complex exhibited marked torque following placement of two lasso sutures around the CTR; they added a third for better fixation stability. However, neither of the prior reports mentioned all of the essential variables that are necessary to achieve the centration required for best performance of a diffractive optic IOL:

1. 1-3-Point fixation $120^{\circ}$ apart.

2. 2-Lasso sutures around only the CTR, not the haptics.

3. 3-Radial orientation of the sutures.

4. 4-Removal of OVD and instillation of a miotic.

5. 5-Setting IOP at physiologic levels.

6. 6-Adjustable tensioning of the three slip knots.

Although the present case did not exhibit complete posterior luxation of the bag complex and pars plana vitrectomy was not required, the same method may be applied under those circumstances. Although the method described herein may be applied in cases with greater degrees of luxation, varying with the specific clinical situation and experience of the surgeon, it might be more prudent to remove the entire capsule bag complex and fixate an IOL to the angle, iris or sclera.

\section{ACKNOWLEDGEMENTS}

Funding. No funding or sponsorship was received for this study or publication of this article.

Authorship. All named authors meet the International Committee of Medical Journal
Editors (ICMJE) criteria for authorship for this article, take responsibility for the integrity of the work as a whole, and have given their approval for this version to be published.

Author Contributions. Samuel Masket performed this study and created all drafts of the manuscript.

Disclosures. Samuel Masket has no conflicts of interest to declare.

Compliance with Ethics Guidelines. Patient consent was received for the publication of this data.

Data Availability. Data sharing is not applicable to this article as no datasets were generated or analyzed during the current study.

Open Access. This article is licensed under a Creative Commons Attribution-NonCommercial 4.0 International License, which permits any non-commercial use, sharing, adaptation, distribution and reproduction in any medium or format, as long as you give appropriate credit to the original author(s) and the source, provide a link to the Creative Commons licence, and indicate if changes were made. The images or other third party material in this article are included in the article's Creative Commons licence, unless indicated otherwise in a credit line to the material. If material is not included in the article's Creative Commons licence and your intended use is not permitted by statutory regulation or exceeds the permitted use, you will need to obtain permission directly from the copyright holder. To view a copy of this licence, visit http://creativecommons.org/licenses/by$\mathrm{nc} / 4.0 /$.

\section{REFERENCES}

1. Ashena Z, Maqsood S, Ahmed SN, Nanavaty MA. Effect of intraocular lens tilt and decentration on visual acuity, dysphotopsia and wavefront aberrations. Vision (Basel). 2020;4(3):41. 
2. Dong J, Wang XL, Deng M, Wang XG. Three-dimensional reconstruction and swept-source optical coherence tomography for crystalline lens tilt and decentration relative to the corneal vertex. Transl Vis Sci Technol. 2021;10(9):13.

3. Ahmed II, Chen SH, Kranemann C, Wong DT. Surgical repositioning of dislocated capsular tension rings. Ophthalmology. 2005;112(10):1725-33.

4. Masket S, Belani S. Combined preoperative topical atropine sulfate $1 \%$ and intracameral non-preserved epinephrine hydrochloride 1:2500 for management of intraoperative floppy-iris syndrome. J Cataract Refract Surg. 2007;33(4):580-2.

5. Fine IH, Hoffman RS, Packer M. Timing of CTR implantation. J Cataract Refract Surg. 2006;32(7): 1075.
6. Moreno-Montanes J, Sanchez-Tocino H, RodriguezCondo R. Complete anterior capsule contraction after phacoemulsification with acrylic intraocular lens and endocapsular ring implantation. J Cataract Refract Surg. 2002;28:717-9.

7. Koçak Altıntaş AG, Omay AE, Çelik S. Spontaneous late intraocular lens and capsule tension ring dislocation. Turk J Ophthalmol. 2017;47(2):106-9.

8. Al-Mezaine HS. Capsule contraction syndrome despite capsular tension ring. Saudi J Ophthalmol. 2007;21:35-7.

9. Deka S, Deka A, Bhattacharjee H. Management of posteriorly dislocated endocapsular tension ring and intraocular lens complex. J Cataract Refractive Surg. 2006;32(5):887-9. 